\title{
Produção de conhecimento sobre avaliação educacional publicada no Brasil durante a década de 1980
}

\author{
The production of knowledge about educational evaluation \\ published in Brazil during the 1980s
}

\author{
Regilson Maciel Borges ${ }^{1}$ \\ ${ }^{1}$ Universidade Federal de Lavras | Departamento de Educação \\ Lavras | MG | Brasil. Contato: regilsonborges@gmail.com \\ http://orcid.org/0000-0001-6115-364X \\ José Carlos Rothen ${ }^{2}$ \\ ${ }^{2}$ Universidade Federal de São Carlos | Centro de Educação e Ciências Humanas| \\ Departamento de Educação \\ São Carlos | SP | Brasil. Contato: josecarlos@ rothen.pro.br \\ http://orcid.org/0000-0002-5360-1913
}
Resumo: O artigo apresenta mapeamento da produção de conhecimento sobre avaliação educacional publicada no Brasil durante a década de 1980 . O referencial teórico e metodológico se baseou na literatura que discute os procedimentos para a coleta de dados em estudos bibliométricos. Os resultados fornecem a ideia do estado da arte da evolução desse campo de conhecimento, bem como permitem algumas constatações a respeito da construção do campo da avaliação no Brasil, evidenciando, por exemplo, o local em que se encontra esse campo e quais os principais autores que o formam.

Palavras-chave: Avaliação educacional. Análise bibliométrica. Produção de conhecimento.

Abstract: The article presents a mapping of the production of knowledge about educational evaluation published in Brazil during the 1980s. The theoretical and methodological framework was based on the literature that discusses the procedures for data collection in bibliometric studies. The results provides the idea of the state of the art of the evolution of this field of knowledge, as well as allow some observations about the construction of the field of evaluation in Brazil, showing, for example, the place of this field and the main authors which form it.

Key words: Educational evaluation. Bibliometric analysis. Knowledge production.

DOI: http://dx.doi.org/10.1590/S1414-40772019000200005

Este é um artigo publicado em acesso aberto (Open Access) sob a licença Creative Commons Attribution Non-Commercial, que permite uso, distribuição e reprodução em qualquer meio, sem restrições desde que sem fins comerciais e que o trabalho original seja corretamente citado. https://creativecommons.org/licenses/by-nc/4.0/ 


\section{Introdução}

$\mathrm{O}$ artigo apresenta os resultados de pesquisa realizada a partir do levantamento da produção científica sobre avaliação educacional publicada no Brasil ao longo dos anos 1980 (BORGES, 2017). O estudo foi realizado em função do que se pretendeu, “[...] o mapeamento da literatura de uma área específica de conhecimento [...]” e a "[...] produção de indicadores bibliométricos [...]" (HAYASHI, 2013, p. 90).

O mapeamento dos dados coletados em diferentes bancos de dados disponíveis online permitiu a identificação do tipo de produção, do editor do texto, da temporalidade dos trabalhos, dos autores, da vinculação institucional e da origem geográfica dos textos. De acordo com Hayashi et al. (2008), esses indicadores permitem conhecer a evolução de um determinado campo de conhecimento, no caso dessa pesquisa, trata-se do campo da avaliação educacional.

Ao considerar e aprofundar os indicadores das produções bibliográficas, os dados forneceram uma ideia do estado da arte da evolução desse campo de conhecimento no Brasil, assim como permitem algumas constatações a respeito da construção do campo científico da avaliação no país na década de 1980. Evidencia, por exemplo, o local onde se encontra esse campo, quais os principais autores que o formam, em decorrência de suas contribuições teóricas sobre a temática da avaliação educacional, assim como indica a quantidade e os tipos de produções publicadas por esses autores no referido período.

A escolha do período pesquisado deu-se em razão do entendimento de que foi durante os anos 1980 que a avaliação acabou recebendo considerável atenção de pesquisadores da área educacional, momento em que são organizadas e publicadas as principais obras sobre a temática da avaliação, que acabaram se constituindo como referências para o campo em construção (SAUL, 2010). Assim também como foi nesse período que se iniciaram as primeiras experiências sistemáticas e propostas para a avaliação das instituições de educação brasileira, tanto para a educação básica quanto para a educação superior (ROTHEN; BARREYRO, 2011a).

A avaliação educacional está sendo compreendida enquanto um campo de conhecimento complexo e polissêmico, com múltiplas e heterogêneas referências, conforme ressalta Dias Sobrinho (2002). Assim, ao nos referirmos à avaliação educacional, estamos atentos a sua complexidade, bem como a sua aplicação a vários objetos, desde a sua forma mais restrita, caso da avaliação da aprendizagem dos alunos, a sua visão mais ampla, caso da avaliação das instituições educacionais, incluindo seus programas, projetos e currículos, e da avaliação dos sistemas e redes de ensino como um todo.

Ainda de acordo com Dias Sobrinho (2002, p. 15-16): 
O campo também se refere a comunidades que se dedicam a investigar a temática e a pôr em prática ações de múltiplos significados e efeitos. No que diz respeito à avaliação, os efeitos, dentre outros, têm sentidos pedagógicos e políticos. Faz também pensar visões de mundo, crenças, atitude e práticas mais ou menos compartilhadas por grupo de cientistas, [...] com profundas disputas e contradições entre grupos e comunidades de intelectuais, profissionais de distintas categorias e organizações políticas com diferentes interesses e concepções. [...].

Deste modo, as noções de avaliação educacional e campo estão sendo tomadas diante do cruzamento de forças que os atravessam e se entrecruzam, fazendo do campo da avaliação educacional uma rede de relações constituída por um “[...] conjunto de formas, manifestações, ideias, grupos, instâncias etc. [...]" que “[...] contribui para dar forma e sentido a uma determinada realidade, e esta age sobre o campo da avaliação, colaborando para sua constituição dinâmica, ou seja, intervindo nas formas e sentidos que historicamente ela adquire" (DIAS SOBRINHO, 2002, p. 16).

Igualmente Stremel (2017) salienta que para seu funcionamento um campo precisa de agentes, instituições, ideias e interesses específicos que lhe dê sustentação e legitimação. Nesse sentido, o estudo da constituição do campo " [...] envolve considerar como fonte aquilo que estrutura os campos, que são as instituições, os agentes, bem como o que se gera no interior dos campos, que são as produções dos agentes, as suas publicações.” (STREMEL, 2017, p. 7). Isso justifica um estudo da natureza apresentada nesse trabalho que se propõe mapear as publicações produzidas por autores que estiveram envolvidos com a temática da avaliação durante os anos 1980. Num período em que a avaliação meramente tecnicista passou a ser questionada, emergindo uma visão da dimensão política da avaliação educacional, como contraponto ao tecnicismo na educação (CALDERÓN; BORGES, 2013a).

O trabalho está organizado em três seções. Na primeira descreve-se o percurso e o desenvolvimento metodológico para a coleta de dados em estudos bibliométricos. Na segunda apresentam-se os resultados do estudo bibliométrico que analisou a produção científica sobre avaliação publicada na década de 1980 em diferentes fontes. Na terceira encontram-se nossas considerações finais.

\section{Metodologia da pesquisa}

O mapeamento da produção científica brasileira sobre avaliação educacional foi analisada a partir de estudo bibliométrico que possibilitou definir o local onde se encontra esse campo e quais são os agentes que dele fizeram parte nos anos 1980. 
A coleta de dados seguiu algumas das etapas metodológicas apontadas por Hayashi (2013) quando o interesse é a construção de indicadores bibliométricos. Dentre as etapas propostas pelo autor se encontram:

a) Escolha das bases de dados: para esse estudo as bases de dados selecionadas foram os sites indexadores de periódicos científicos ${ }^{1}$, os sites dos Programas de Pós-Graduação em Educação (PPGE) ${ }^{2}$, os sites das bibliotecas das instituições a que se vinculam os programas e o Portal Domínio Público ${ }^{3}$.

b) Identificação das fontes: para essa identificação foram utilizados os seguintes descritores: “Avaliação”, “Avaliar”, “Avaliação Educacional”, “Aprovação”, "Reprovação”, "Vestibular", "Exames vestibulares", "Qualidade (da educação e do ensino)", "Rendimento escolar", "Fracasso escolar", "Sucesso escolar", "Provas" e "Evasão". Essa diversidade de descritores se deu em razão da tentativa de abarcar o maior número possível de trabalhos, para isso consideramos os diferentes modos referenciados pela literatura que analisa a produção científica brasileira a respeito da avaliação (VIANNA, 1992; CANDAU; OSWALD, 1995).

c) Organização do material coletado: o material selecionado foi organizado em planilha Microsoft Excel contendo os dados principais de cada produção, como "Tipo de Produção", "Revista/Editora/IES”, “Ano”, "Volume” e "Número", "Seção na Revista”, “Autor”, "Vínculo Institucional", “Orientador", “Título do Trabalho" e "Palavras-chave dos trabalhos".

d) Modelagem dos dados: nessa etapa buscou-se eliminar nas planilhas Microsoft Excel organizadas as inconsistências dos dados coletados, como erro de grafia, duplicidades, eliminação de campo em branco, entre outros que podem atrapalhar a elaboração de tabelas e gráficos.

e) Confecção de gráficos e tabelas dinâmicas: nesta última etapa, após organização e modelagem do material coletado, foi possível a criação de tabelas dinâmicas que permitiram visualizar a distribuição do conhecimento sobre avaliação ao longo de toda a década de 1980.

1 Disponíveis nos sites do Scientific Electronic Library Online (disponível em: http://www.scielo.br/scielo.php?script=sci_subject\&lng=pt); e do Educ@ Publicações online de educação (disponível em: http://educa.fcc.org.br/scielo.php?script=sci_alphabetic\&lng=pt\&nrm=iso).

2 Foram visitados os sites dos 29 programas de mestrado e doutorado em educação, existentes desde os anos 1960, conforme assinalam Fávero (1993) e Warde (1993): ISAE-FGV; PPGE-PUCRJ; PPGE-PUCRS; PPGE-PUCSP; PPGE-UCP; PPGE-UERJ; PPGE-UFAM; PPGE-UFBA; PPGE-UFC; PPGE-UFES; PPGE-UFF; PPGE-UFG; PPGE-UFMG; PPGE-UFMS; PPGE-UFMT; PPGE-UFPB; PPGE-UFPE; PPGE-UFPR; PPGE-UFRGS; PPGE-UFRJ; PPGE-UFRGN; PPGE-UFSCAR; PPGE-UFSC; PPGE-UFSM; PPGE-UNB; PPGE-UNESP; PPGE-UNICAMP; PPGE-UNIMEP; PPGE-USP.

${ }^{3}$ Disponível em: http://www.dominiopublico.gov.br/pesquisa/PesquisaObraForm.jsp 
f) Análise e interpretação dos dados: que foi feita à luz análise bibliométrica e das teorias que fundamentaram a pesquisa, no caso dessa pesquisa do referencial teórico da noção de campo científico de Pierre Bourdieu.

No levantamento foram encontrados um total de 595 publicações, distribuídas em 6 capítulos de livros, 7 documentos oficiais, 9 publicações em anais de eventos, 65 livros, 252 artigos científicos, 244 dissertações e 12 teses (10 de doutorado e 2 de professor titular). Todo esse material encontra-se organizado em planilhas Microsoft Excel contendo os principais dados de cada produção, como: tipo de produção; revista/editora/IES; ano, volume e número; seção na revista; autor; vínculo institucional; orientador; título do trabalho; palavras-chave dos trabalhos e nível de ensino (BORGES, 2017).

\section{Mapeamento da produção sobre avaliação educacional (1980-1989)}

A seguir são apresentados dados coletados e que possibilitam uma visão panorâmica da produção levantada no período de 1980 a 1989.

No Gráfico 1 os 595 trabalhos encontrados são distribuídos segundo o ano de sua publicação, nele se observa a variação da produção sobre a temática analisada, concentrada, principalmente, nos anos de 1980, que obteve 72 trabalhos catalogados e em 1983, com 77 trabalhos, com um queda considerável nos anos de 1985 e 1989, tendo sido produzido 45 trabalhos nesses dois anos.

GRÁFICO 1 - Distribuição do material por ano de publicação (1980-1989)

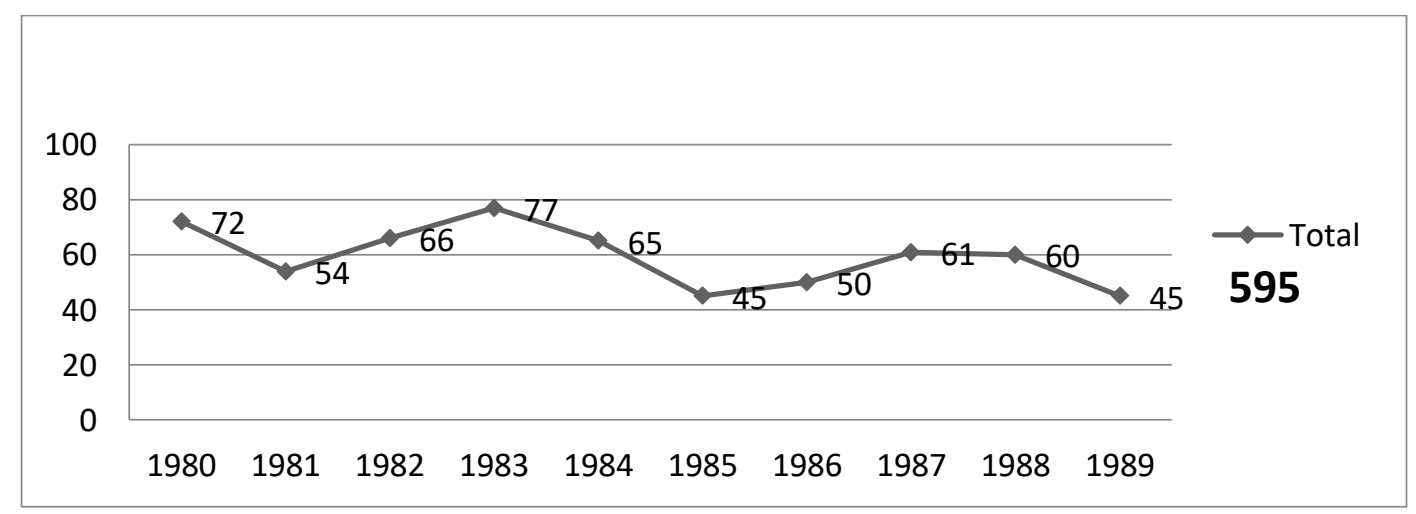

Fonte: elaboração dos autores a partir das publicações encontradas (2015)

\footnotetext{
${ }^{4}$ Esse material completo está apresentado nos Apêndices do relatório final da pesquisa sob a forma da Tese de Doutorado que se encontra disponível online no Repositório Institucional UFSCar. Disponível em: https://repositorio.ufscar.br/handle/ufscar/9046.
} 
A maior concentração da produção científica sobre avaliação na primeira metade da década de 1980 no Brasil, conforme aponta o Gráfico 1, resulta principalmente da quantidade de artigos científicos e das dissertações e teses elaboradas sobre o tema da avaliação. A totalidade da produção publicada no período analisado (595 trabalhos) também mostra o interesse de pesquisadores brasileiros da área da educação em abordar questões sobre avaliação educacional na referida década. Trata-se de um período em que, segundo Saviani (2013, p. 402), as pedagogias críticas buscam orientar a prática educativa brasileira, com "[...] teorias que não apenas se constituíssem como alternativa a pedagogia oficial, mas que a ela se contrapusessem.”. No caso específico da avaliação, Saul (2010) menciona que, nesse período, os autores brasileiros que atuavam na área da avaliação começaram a se preocupar em discutir e aprofundar a avaliação numa abordagem de natureza mais qualitativa.

Na Tabela 1 seguinte, os estudos encontram-se distribuídos segundo o tipo de produção (artigo de revista, anais de evento, capítulo de livro, dissertação de mestrado, documento oficial, livro, tese de doutorado e tese de professor titular). Nela é possível observar que, no período analisado, há grande publicação de artigos científicos e dissertações de mestrado sobre avaliação. Cabe salientar que esses dois tipos de produções, juntamente com as teses, são, segundo Soares (1989), as que melhor expressam o conhecimento ainda em construção, cuja circulação ocorre de forma concomitante à sua elaboração, o que nos possibilita uma dimensão das temáticas privilegiadas em determinados períodos.

TABELA 1 - Distribuição do material por tipo de produção (1980-1989)

\begin{tabular}{cc}
\hline Tipos de Trabalho & Quantidade \\
\hline Artigo de revista & 252 \\
Dissertação de Mestrado & 243 \\
Livro & 65 \\
Tese de Doutorado & 10 \\
Anais de Evento & 9 \\
Documento Oficial & 8 \\
Capítulo de Livro & 6 \\
Tese de Professor Titular & 2 \\
Total geral & $\mathbf{5 9 5}$ \\
\hline
\end{tabular}

Fonte: elaboração dos autores (2015)

Ainda hoje os artigos publicados em revistas cientificas junto com as teses de doutorado e dissertações de mestrado ocupam lugar de destaque naquilo que Meadows (1999, p. vii) chama de coração da ciência: a comunicação científica, pois, segundo o autor ' [...] a esta não 
cabe reivindicar com legitimidade este nome enquanto não houver sido analisada e aceita pelos pares. Isto exige, necessariamente, que seja comunicada”. Neste processo as revistas científicas ocupam um papel de grande relevância na difusão e consolidação da ciência, tendo em vista que a pesquisa para reivindicar este nome precisa passar pelo crivo e aceitação da comunidade científica, e nesse processo as revistas acabam sendo um dos produtos com maior aceitação como registro de produção (STUMPF, 2003).

Na Tabela 2 são identificadas as revistas científicas que tiveram suas produções catalogadas no levantamento realizado, nela se encontram a quantidade de artigos produzidos de acordo com o ano de sua publicação:

TABELA 2 - Distribuição do material por revistas científicas e anos (1980-1989)

\begin{tabular}{cccccccccccc}
\hline Revistas & $\mathbf{1 9 8}$ & $\mathbf{1 9 8}$ & $\mathbf{1 9 8}$ & $\mathbf{1 9 8}$ & $\mathbf{1 9 8}$ & $\mathbf{1 9 8}$ & $\mathbf{1 9 8}$ & $\mathbf{1 9 8}$ & $\mathbf{1 9 8}$ & $\mathbf{1 9 8}$ & $\begin{array}{c}\text { Total } \\
\text { geral }\end{array}$ \\
\hline Educação e Seleção & 13 & 16 & 14 & 18 & 14 & 14 & 20 & 17 & 14 & 7 & 147 \\
Cadernos de Pesquisa & 5 & 2 & 2 & 13 & 13 & 4 & 5 & 4 & 2 & 6 & 56 \\
Em Aberto & & & 6 & & & & 1 & 3 & 2 & 3 & 15 \\
Educação e Avaliação & 8 & 5 & & & & & & & & & 13 \\
Educação \& Sociedade & & 1 & & & 2 & & & 3 & 1 & 1 & 8 \\
$\quad \begin{array}{c}\text { Revista da } \\
\text { Fac.Edu.USP }\end{array}$ & & & & & & & & 2 & & 1 & 5 \\
Educação em Revista & & & & & & 1 & 2 & 2 & & & 5 \\
Cadernos CEDES & & & & & 1 & & & & 2 & & 3 \\
Total geral & $\mathbf{2 6}$ & $\mathbf{2 4}$ & $\mathbf{2 4}$ & $\mathbf{3 1}$ & $\mathbf{3 0}$ & $\mathbf{1 9}$ & $\mathbf{2 8}$ & $\mathbf{3 1}$ & $\mathbf{2 1}$ & $\mathbf{1 8}$ & $\mathbf{2 5 2}$ \\
\hline
\end{tabular}

Fonte: elaboração dos autores (2015)

A Tabela 2 mostra que a maioria dos artigos foi publicada por duas revistas editadas por uma fundação especializada na área da avaliação, a Fundação Carlos Chagas (FCC), são elas: "Educação e Seleção", que aparece com 147 artigos, e "Cadernos de Pesquisa", com 56 artigos. Trata-se de uma instituição que tem um longo histórico com questões relacionadas à seleção, medida e avaliação educacional, tal como aponta Sousa (2005) ao analisar os 40 anos de contribuição da FCC à avaliação educacional, no período de 1972 a 2003. Nessa instituição se encontraram vinculados pesquisadores que há algum tempo realizaram estudos nessa área de conhecimento, dentre os quais estão Adolpho Ribeiro Netto, Dagmar M. L. Zibas, Bernadete A. Gatti, Heraldo Marelim Vianna, entre outros autores listados mais a frente na Tabela 6.

Sobre a revista Educação e Seleção, Calderón e Borges (2013b, p. 171) mencionam que se trata de uma das "revistas precursoras" na área dos estudos em avaliação educacional, que circulou de 1980 a 1989, e juntamente com a revista Educação e Avaliação, editada em dois 
únicos números pela Cortez, em 1980 e 1981, possibilitaram o aparecimento de outras revistas especializadas no tema da avaliação. Os autores ressaltam ainda a existência de outras importantes publicações brasileiras que "apesar de não se vocacionarem especificamente à temática avaliação, também a abordam em seu conteúdo de forma esporádica” (CALDERÓN; BORGES, 2013b, p. 172), dentre as quais se incluem os Cadernos de Pesquisa da FCC.

Na Tabela 3, as 12 teses e 243 dissertações encontradas são distribuídas de acordo com o ano de sua defesa. Nela pode-se observar que nos anos de 1980, 1982 e 1983 a produção ultrapassa o total de 30 publicações, obtendo menor concentração nos anos de 1985, 1987 e 1989, com variação de 17 e 19 trabalhos.

TABELA 3 - Distribuição do material por ano e tipo de estudo (dissertação ou tese) (1980-1989)

\begin{tabular}{ccccc}
\hline Ano & Dissertação de Mestrado & Tese de Doutorado & Tese de Professor Titular & Quantidade \\
\hline 1980 & 32 & & 2 & 32 \\
1981 & 22 & 2 & & 26 \\
1982 & 32 & & 32 \\
1983 & 35 & 3 & 38 \\
1984 & 28 & 1 & 29 \\
1985 & 16 & 1 & 17 \\
1986 & 14 & & 14 \\
1987 & 19 & & & 19 \\
1988 & 29 & & & 29 \\
1989 & 16 & 3 & $\mathbf{2}$ & 19 \\
Total geral & $\mathbf{2 4 3}$ & $\mathbf{1 0}$ & $\mathbf{2 5 5}$ \\
\hline
\end{tabular}

Fonte: elaboração dos autores (2015)

Os dados da Tabela 3 corroboram a análise do Gráfico 1 apresentado no início desta seção, que apontou a concentração desse tipo de produção nos anos iniciais da década de 1980. Conforme mencionado, foi um período em que o campo educacional brasileiro foi marcado pela intensa mobilização e organização, pela considerável ampliação da produção acadêmicocientífica e pela busca de teorias educacionais que mais se adequassem aos anseios daquele momento (SAVIANI, 2013). Nosella (2010) destaca que a ampliação da produção acadêmicocientífica sobre educação nos anos 1980 seu deu pela consolidação e expansão dos Programas de Pós-Graduação em Educação no período, ocasionando o desenvolvimento de um pensamento crítico, a ampliação das linhas de pesquisa, a diversificação teórico-metodológica e a utilização de fontes variadas na pesquisa.

$\mathrm{Na}$ Tabela 4 são apresentadas as Instituições de Ensino Superior (IES) onde foram defendidas as teses e dissertações no período pesquisado, com destaque para a Universidade 
Federal do Rio de Janeiro (UFRJ) com 79 dissertações defendidas em seu Programa de PósGraduação em Educação (PPGE), que teve início em 1972, com a criação do Mestrado, e se ampliou em 1980, com a criação do Doutorado. Mais de um terço dessa produção foi orientada por duas professoras vinculadas ao programa: Ligia Gomes Elliot, que orientou 17 trabalhos, e Lilia da Rocha Bastos, que orientou 15 trabalhos.

TABELA 4 - Distribuição dos trabalhos (teses e dissertações) por instituição (1980-1989)

\begin{tabular}{|c|c|}
\hline IES & Quantidade \\
\hline UFRJ & 79 \\
\hline PUC-SP & 17 \\
\hline UERJ & 17 \\
\hline UNB & 16 \\
\hline UFES & 15 \\
\hline UFSCAR & 13 \\
\hline PUC-RJ & 11 \\
\hline UFPR & 11 \\
\hline UFF & 10 \\
\hline UNIMEP & 9 \\
\hline FGV & 8 \\
\hline PUC-RS & 8 \\
\hline UFRGS & 8 \\
\hline USP & 8 \\
\hline UFSM & 7 \\
\hline UNICAMP & 6 \\
\hline UFBA & 5 \\
\hline UFC & 4 \\
\hline UFMG & 2 \\
\hline UFRN & 1 \\
\hline Total geral & 255 \\
\hline
\end{tabular}

Fonte: elaboração dos autores (2015)

A constatação da UFRJ como instituição onde foram defendidas a maioria das teses e dissertações merece destaque, na medida em que outros estudos realizados na perspectiva de estado da arte em torno da produção científica sobre avaliação educacional em diversas fontes, tais como artigos de revistas científicas, dissertações, teses, livros, internet e anais de eventos (VIANNA, 1992; CANDAU; OSWALD， 1995; SOUSA, 1994a; 1994b; 1995; 1996; BARRETTO; PINTO, 2001; BARRETTO; PINTO; MARTINS; DURAN, 2001; GONÇALVES FILHO, 2003; SOUSA, 2005; TEIXEIRA, 2006; GAMA; COELHO; CAMELO, 2007; FREZZA; SILVA, 2009; ULER, 2010; ROTHEN; BARREYRO, 2011b; 
TAVARES; OLIVEIRA; SEIFFERT, 2011; BORGES; CALDERÓN, 2011; POLTRONIERI; CALDERÓN, 2012; SOUSA, 2012; SILVA, 2013), a UFRJ não aparece com o mesmo destaque que teve em nosso levantamento. Apenas os trabalhos de Sousa (1994a; 1996), sobre a natureza e contribuições das pesquisas sobre Avaliação da Aprendizagem no Brasil no período de 1980 a 1990, mencionam a UFRJ como uma das quatro universidades do país com maior número de trabalho sobre o tema pesquisa pela autora.

$\mathrm{Na}$ Tabela 5 foram identificadas as editoras que publicaram os livros e capítulos de livros produzidos durante os anos 1980. Destaque para a Editora Cortez, com seis produções; Editora da UFRGS, com cinco produções; Editora Pedagógica Universitária (EPU), com quatro produções; e Ministério da Educação e Editora Vozes, com três produções cada. Saviani (2013, p. 407), menciona que foi nesse período, de 1980 a 1991, que “[...] as principais editoras criaram coleções de educação, abrindo-se, inclusive, editoras especializadas na área.”.

TABELA 5 - Distribuição dos livros e capítulos de livros por editora (1980-1989) (continua)

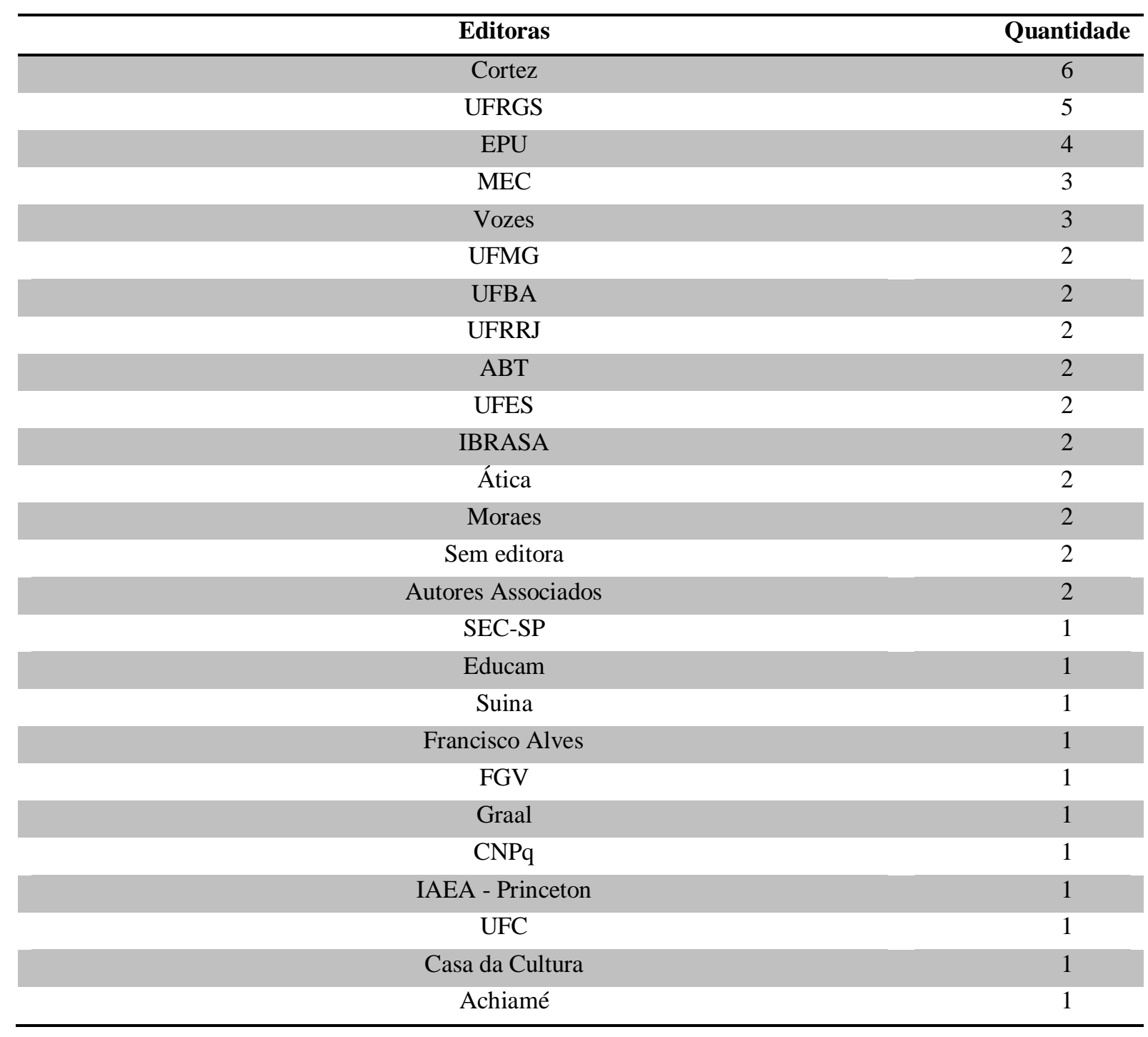




\begin{tabular}{cc}
\hline INEP & 1 \\
SEC-BA & 1 \\
UNICAMP & 1 \\
Segrase & 1 \\
Zahar & 1 \\
SME-Juiz de Fora & 1 \\
UNB & 1 \\
Brasiliense & 1 \\
ALMED & 1 \\
CRUB & 1 \\
Mestre Jou & 1 \\
UFPR & 1 \\
CEDATE & 1 \\
FCC & 1 \\
Pallotti & 1 \\
Pioneira & 1 \\
ABMES & 1 \\
IUPERJ & 1 \\
LTC & 1 \\
Total geral & 1 \\
\hline & $\mathbf{7 2}$ \\
\hline
\end{tabular}

Fonte: elaboração dos autores (2015)

A respeito das editoras, os trabalhos de Saviani (2013) e Ricardo Filho (2010) tecem considerações sobre a importância e supremacia de editoras no processo de configuração do espaço educacional brasileiro, contando, inclusive, com publicações de coletâneas específicas para a área da educação, sob a responsabilidade de renomados autores. Ricardo Filho (2010) observa ainda que muitos desses autores, como Antonio Joaquim Severino, Casemiro dos Reis Filho, Dermeval Saviani, Joel Martins, Moacir Gadotti, dentre outros, além de fazerem parte do corpo editorial de editoras como Cortez e Moraes, também estavam vinculados a programas de pós-graduação em educação considerados como os mais influentes no final da década de 1970 no Brasil.

Na Tabela 6 são identificados os autores que tiveram mais de duas publicações durante o período analisado, com destaque para os onze autores vinculados a Fundação Carlos Chagas (FCC); os nove vinculados a Pontifícia Universidade Católica do Rio de Janeiro (PUC-RJ); os seis vinculados a Pontifícia Universidade Católica de São Paulo (PUC-SP); os seis vinculados a Universidade Federal do Rio Grande do Sul (UFRGS); os seis vinculados a Universidade de Brasília (UNB); os seis vinculados a Universidade de São Paulo (USP); os três vinculados a Universidade Federal de Pernambuco (UFPE); os três vinculados ao Serviço Nacional de 
Aprendizagem Industrial (SENAI-SP); e os três vinculados a Universidade Federal do Ceará (UFC).

TABELA 6 - Distribuição dos autores que produziram mais de dois textos (1980-1989) (continua)

\begin{tabular}{|c|c|c|}
\hline Autor & Vínculo Institucional & Quantidade \\
\hline Heraldo Marelim VIANNA & FCC & 28 \\
\hline Bernardete A. GATTI & FCC/PUC-SP & 14 \\
\hline Menga LÜDKE & PUC-RJ & 12 \\
\hline Adolpho RIBEIRO NETTO & FCC & 10 \\
\hline Nícia Maria BESSA & PUC-RJ & 10 \\
\hline Zélia D. MEDIANO & PUC-RJ & 10 \\
\hline Bernardo BUCHWEITZ & UFRGS & 9 \\
\hline Pedro DEMO & UNB/IPEA/INEP & 8 \\
\hline Maria Amélia Azevedo GOLDBERG & FCC/PUC-SP & 8 \\
\hline Fernando Lang da SILVEIRA & UFRGS/PUC-RS & 7 \\
\hline Leny Campagno Cyrino BASSO & PUC-RJ & 7 \\
\hline Sérgio Costa RIBEIRO & CESGRANRIO/PUC-RJ & 7 \\
\hline Ana Maria de Oliveira LOPES & PUC-RJ & 6 \\
\hline Dagmar M. L. ZIBAS & FCC & 6 \\
\hline Giselda Santana MORAIS & UFS & 6 \\
\hline Marco Antônio MOREIRA & UFRGS & 6 \\
\hline Elba Siqueira de Sá BARRETTO & FCC & 5 \\
\hline Léa DEPRESBITERIS & SENAI-SP & 5 \\
\hline Maria Lúcia Faria MORO & UFPR & 5 \\
\hline Maria do Socorro Taurino BRITO & UFPB & 5 \\
\hline Cláudio de Moura CASTRO & CAPES/IPEA & 4 \\
\hline Lúcia Marques PINHEIRO & INEP & 4 \\
\hline Zaia BRANDÃO & IUPERJ & 4 \\
\hline Anna Maria Bianchini BAETA & FGV & 3 \\
\hline Analúcia Dias SCHLIEMANN & UFPE & 3 \\
\hline Any Dutra Coelho da ROCHA & UCAM & 3 \\
\hline Aida Maria MONTEIRO & UFPE & 3 \\
\hline Carlos Alberto Serpa de OLIVEIRA & CESGRANRIO/PUC-RJ & 3 \\
\hline Clarilza Prado de SOUSA & PUC-SP & 3 \\
\hline Flávia de Barros CARONE & USP & 3 \\
\hline Heloísa LÜCK & UFPR & 3 \\
\hline Jussara Maria Lerch HOFFMAN & UFRGS & 3 \\
\hline Lauro MORHY & UNB & 3 \\
\hline Lygia Correa Dias de MORAES & USP & 3 \\
\hline Maria Laura P. B. FRANCO & FCC/PUC-SP & 3 \\
\hline Marli E. D. A. ANDRÉ & PUC-RJ & 3 \\
\hline Raimundo Alberto NORMANDO & UFC & 3 \\
\hline Simon SCHWARTZMAN & IUPERJ/FGV & 3 \\
\hline Alda BALTAR & UNB & 2 \\
\hline Alaor Correa PINTO & SENAI-SP & 2 \\
\hline
\end{tabular}




\begin{tabular}{|c|c|c|}
\hline Ana Maria SAUL & PUC-SP & 2 \\
\hline Cipriano LUCKESI & UFBA & 2 \\
\hline Daniel Augusto MOREIRA & USP & 2 \\
\hline Enilde L. J. FAULSTICH & UNB & 2 \\
\hline Ênio de MAGALHÃES & UNB & 2 \\
\hline Eny Marisa MAIA & FCC & 2 \\
\hline Ernesto SCHIEFELBEIN & $\mathrm{ONU}$ & 2 \\
\hline Fúlvia ROSEMBERG & FCC/PUC-SP & 2 \\
\hline Guiomar Namo MELLO & FCC/PUC-SP & 2 \\
\hline José Geraldo TEIXEIRA & UFJF & 2 \\
\hline José Luiz Pieroni RODRIGUES & SENAI-SP & 2 \\
\hline Lauro Amélio PATZLAFF & UNB & 2 \\
\hline Lucila Maria Costi SANTAROSA & UFRGS & 2 \\
\hline Luiz PASQUALI & $\mathrm{UNB} / \mathrm{MEC}$ & 2 \\
\hline Manuel Luiz LEÃO & UFRGS & 2 \\
\hline Maria Aparecida Ciavatta FRANCO & UERJ/UFF & 2 \\
\hline Maria Helena Souza PATTO & USP & 2 \\
\hline Maria do Horto Soares MOTTA & UFRGS & 2 \\
\hline Maria Lúcia Faria MORA & USP & 2 \\
\hline Maria Thereza Fraga ROCCO & UFF & 2 \\
\hline Maria Lúcia Lopes DALLAGO & UFC & 2 \\
\hline Mauro Villar de QUEIROZ & UFC & 2 \\
\hline Michel THIOLLENT & UFRJ & 2 \\
\hline Ruben KLEIN & $\mathrm{UFRJ} / \mathrm{CNPq}$ & 2 \\
\hline Robert STAKE & Universidade de Illinois & 2 \\
\hline Rose Neubauer da SILVA & FCC & 2 \\
\hline Sueli Cotrim TENCA & USP & 2 \\
\hline Terezinha Nunes CARRAHER & UFPE & 2 \\
\hline Thereza PENNA FIRME & UFRGS/PUC-RJ/UFRJ & 2 \\
\hline UFC & & 2 \\
\hline Wilma Porto de PRIOR & UFS & 2 \\
\hline
\end{tabular}

Fonte: elaboração dos autores (2016)

De um total de 548 autores que publicaram ao longo da década de 1980, 71 tiveram mais de duas publicações. Nesse quadro destacamos a presença de Robert Stake, reconhecido autor estadunidense da avaliação, como um dos autores que produziram mais de dois textos sobre a temática no Brasil. Isso se deve a própria dimensão do levantamento bibliográfico realizado, que não se restringiu, num primeiro momento, apenas a autores brasileiros, mas a toda produção de conhecimento sobre o tema da avaliação publicada na década de 1980 no país. Nessa lista se incluem autores de outras nacionalidades, como Alain Bienayme (França), Cayetano De Lella (México), Denis C. Phillips (Estados Unidos), dentre outros que publicaram um trabalho no período pesquisado. 
Dos 71 autores que publicaram mais de dois trabalhos, procuramos selecionar aqueles que pudessem ser considerados os mais relevantes para se pensar a construção de um campo científico brasileiro da avaliação. Para tanto fizemos um levantamento de trabalhos que analisam a trajetória histórica da avaliação educacional no Brasil (PENNA FIRME, 1988; VIANNA, 1992; SOUSA, 1998; DEPRESBITERIS, 1999; GATTI, 2002; 2009; SOUSA, 2005; FREITAS, 2007; BARREYRO; ROTHEN, 2008; SAUL, 2010), com a intenção de verificar a presença de alguns dos autores listados na Tabela 6. Do cruzamento das informações contidas nesses trabalhos, juntamente com os resultados encontrados no estudo quantitativo que realizamos, chegamos a alguns nomes que estamos considerando como os principais autores da avaliação educacional na década de 1980 no Brasil.

Assim, dos 71 autores que foram listados na Tabela 6, 19 deles também aparecem com destaque nos trabalhos listados acima, em razão de suas contribuições teóricas e interesse pela temática da avaliação educacional. Os 19 autores são apresentados no Quadro 1 a seguir, que identifica esses autores e suas respectivas vinculações institucionais à época.

QUADRO 1 - Os principais autores da avaliação educacional na década de 1980

\begin{tabular}{|cc|}
\hline Autores & Vínculo Institucional \\
Adolpho RIBEIRO NETTO & FCC \\
Ana Maria SAUL & PUC-SP \\
Bernardete Angelina GATTI & PUC-SP/FCC \\
Clarilza Prado de SOUSA & PUC-SP \\
Claudio de Moura CASTRO & CAPES/IPEA \\
Cipriano Carlos LUCKESI & UFBA \\
Elba Siqueira de Sá BARRETTO & FCC \\
Heraldo Marelim VIANNA & FCC \\
Jussara Maria Lerch HOFFMAN & UFRGS \\
Lea DEPRESBITERIS & SENAI-SP \\
Maria Amélia Azevedo GOLDBERG & FCC/PUC-SP/UNICAMP \\
Maria Laura Puglisi Barbosa FRANCO & PUC-SP/FCC \\
Marli Eliza Dalmazo Afonso de ANDRÉ & PUC-RJ \\
Menga LÜDKE & PUC-RJ/UFF \\
Pedro DEMO & UNB/IPEA/INEP/OMEP \\
Ruben KLEIN & UFRJ/CNPq \\
Sérgio Costa RIBEIRO & CESGRANRIO/PUC-RJ \\
Simon SCHWARTZMAN & IUPERJ/FGV \\
Thereza PENNA FIRME & UFRGS/PUC-RJ/UFRJ \\
\hline
\end{tabular}

Fonte: elaboração dos autores (2016)

Temos no Quadro 1 uma lista que inclui diferentes autores, vinculados as mais diversas instituições e centros de pesquisa, que se preocuparam principalmente com a formulação e difusão de conhecimento em torno da temática da avaliação educacional no Brasil, são eles: Adolpho Ribeiro Netto (FCC), Ana Maria Saul (PUC-SP), Bernardete Angelina Gatti (PUCSP/FCC), Clarilza Prado de Sousa (PUC-SP), Claudio de Moura Castro (CAPES/IPEA), 
Cipriano Carlos Luckesi (UFBA), Elba Siqueira de Sá Barretto (FCC), Heraldo Marelim Vianna (FCC), Jussara Maria Lerch Hoffmann (UFRGS), Lea Depresbiteris (SENAI-SP), Maria Amélia Azevedo Goldberg (FCC/PUC-SP/UNICAMP), Maria Laura Puglisi Barbosa Franco (PUC-SP/FCC), Marli Eliza Dalmazo Afonso de André (PUC-RJ), Menga Lüdke (PUCRJ/UFF), Pedro Demo (UNB/IPEA/INEP/OMEP), Ruben Klein (UFRJ/CNPq), Sérgio Costa Ribeiro (CESGRANRIO/PUC-RJ), Simon Schwartzman (IUPERJ/FGV) e Thereza Penna Firme (UFRGS/PUC-RJ/UFRJ).

No total são seis autores vinculados a Fundação Carlos Chagas; cinco a Pontifícia Universidade Católica de São Paulo; quatro a Pontifícia Universidade Católica do Rio de Janeiro; dois a Universidade Federal do Rio de Janeiro; dois ao Instituto de Pesquisa Econômica Aplicada; dois a Universidade Federal do Rio Grande do Sul; um a Coordenação de Aperfeiçoamento de Pessoal de Nível Superior; um a Universidade Federal da Bahia; uma ao Serviço Nacional de Aprendizagem Industrial; uma a Universidade Estadual de Campinas; uma a Universidade Federal Fluminense; um a Universidade de Brasília; um ao Instituto Nacional de Estudos e Pesquisas; um ao Conselho Nacional de Desenvolvimento Científico e Tecnológico; um a Cesgranrio, um ao Instituto Universitário de Pesquisas do Rio de Janeiro; um a Fundação Getúlio Vargas; e um a Organização Mundial para Educação Pré-Escolar.

As produções desses autores discutem a avaliação sob duas principais perspectivas: a) uma que discute a avaliação na sua vertente de medidas, testes e indicadores educacionais, com destaque para as produções de Heraldo Vianna, Adolpho Ribeiro Netto, Sérgio Costa Ribeiro, Simon Schwartzman, Claudio de Moura Castro e Ruben Klein; e b) outra que, fundamentada numa abordagem de avaliação mais qualitativa e participativa, passaram a desenvolver referenciais teóricos alternativos ao modelo de avaliação classificatório e excludente predominante no período, com destaque para as produções de Ana Maria Saul, Bernardete Gatti, Cipriano Luckesi, Clarilza Prado de Sousa, Elba Barretto, Jussara Hoffman, Léa Depresbiteris, Maria Amélia Goldberg, Maria Laura Franco, Marli André, Menga Lüdke, Pedro Demo e Thereza Penna Firme.

No primeiro grupo as discussões dos autores se concentram em estudos que associam avaliação a medidas resultantes do desempenho dos estudantes com a aplicação de testes em exames vestibulares e da mensuração da produção científica e acadêmica da pós-graduação brasileira, considerando a avaliação como diagnóstico da realidade educacional brasileira baseada na seletividade da educação, no desempenho escolar e na identificação de problemas que ocorriam nas políticas e programas educacionais. No segundo grupo os autores teorizam sobre a temática da avaliação educacional tendo como referência a literatura da área que 
apresenta diferentes definições e concepções da avaliação educacional e expõem suas experiências como pesquisadores educacionais conhecedores de diferentes métodos qualitativos, assim como relatam suas práticas avaliativas implementadas em programas que focalizam a avaliação da aprendizagem e a avaliação de programas educacionais.

\section{Considerações finais}

O artigo apresentou o mapeamento da produção científica sobre avaliação educacional publicada ao longo da década de 1980 no Brasil, com a intenção de apontar algumas indicações sobre a construção de um campo científico brasileiro da avaliação.

O campo é compreendido como algo dinâmico e histórico, que é construído e reconstruído "[...] à medida que sociedade se torna mais complexa e crescem as comunidades de intelectuais [...]” (DIAS SOBRINHO, 2002, p. 16). Além disso, “[...] o campo é disputado por grupos que são partidários de um determinado enfoque [...]. Cada enfoque tem importantes seguidores, apresenta explicitamente alguns fundamentos racionais, aparece nas bibliografias, é posto em prática [...].” (DIAS SOBRINHO, 2002, p. 17). Disso entende-se o campo enquanto espaço de relações, cujos agentes possuem interesses específicos comuns que estimulam as pessoas e tornam possível o funcionamento do campo num determinado momento histórico (BOURDIEU, 2004).

Nesta pesquisa, a análise desse campo foi realizada a partir dos indicadores da produção de conhecimento sobre avaliação educacional publicada ao longo da década de 1980, por considerarmos que foi nesse período que começou uma reflexão teórica mais aprofundada em torno da avaliação educacional no país, quando também tiveram início as primeiras experiências sistemáticas e propostas de avaliação das instituições de educação brasileira (ROTHEN; BARREYRO, 2011a). Os indicadores bibliométricos dessa produção possibilitaram a identificação do tipo de produção, revista/editora/IES, ano, volume e número, seção na revista, autor, vínculo institucional, orientador, título do trabalho e palavras-chave. $\mathrm{O}$ cruzamento desse material, dentre outros resultados, evidenciou o local em que se encontra esse campo e quais os principais autores que o formaram.

Se considerarmos, por exemplo, o vínculo institucional dos autores que tiveram mais de duas publicações no período analisado (Quadro 1), temos grupos formados em seis instituições e/ou centros de pesquisa, é o caso dos seis autores vinculados a FCC, dos cinco a PUC-SP, dos quatro a PUC-RJ, dos dois IPEA, dos dois a UFRJ e dos outros dois a UFRGS. Excetuando-se

o IPEA, as demais instituições aparecem com frequência nos estudos que analisam o 
conhecimento produzido sobre o tema da avaliação no país, é o que salienta Sousa (1996) ao constatar que a concentração de estudos sobre avaliação da aprendizagem no período de 1980 a 1990 em quatro Universidades: a PUC-SP, a PUC-RJ, a UFRJ e a UFES. Já os trabalhos de Candau e Oswald (1995), Sousa (2005) e Poltronieri e Calderón (2012) mencionam a importância da FCC no que se refere à produção e divulgação do conhecimento científico na área da avaliação.

A partir da afinidade nas suas produções, ou seja, os focos comuns de interesses em determinadas temáticas abordadas e de acordo com seus alvos em jogo e interesses específicos (BOURDIEU, 2004), e segundo as relações igualmente comuns entre os agentes que dão sustentação para o funcionamento do campo, foi também possível classificar os 19 autores identificados nesta pesquisa como os principais autores da avaliação educacional na década de 1980, em dois grupos teóricos. O primeiro grupo, que inclui autores como Heraldo Vianna, Adolpho Ribeiro Netto, Sérgio Costa Ribeiro, Simon Schwartzman, Claudio de Moura Castro e Ruben Klein, discute a avaliação na perspectiva de resultados e apresentam basicamente estudos de cunho quantitativo sobre o rendimento escolar dos alunos que são mensurados por meio de testes de conhecimentos, tal como os aplicados nos exames vestibulares. A avaliação nessa abordagem se confunde com a mensuração do rendimento escolar aferida por meio de testes que possibilitariam medir com rigor a aprendizagem dos alunos, proporcionando-lhes o acesso ao ensino superior, quando do emprego dos exames vestibulares. Por outro lado, as medidas também se traduzem nos indicadores da produção científica da pós-graduação que indicariam o desempenho, isto é, a qualidade, dos cursos de mestrados e doutorados no país.

O outro grupo, que inclui autores como Ana Maria Saul, Bernardete Gatti, Cipriano Luckesi, Clarilza Prado de Sousa, Elba Barretto, Jussara Hoffman, Léa Depresbiteris, Maria Amélia Goldberg, Maria Laura Franco, Marli André, Menga Lüdke, Pedro Demo e Thereza Penna Firme, discute a avaliação na perspectiva de processo, com a presença de estudos de cunho qualitativo que analisam a literatura da avaliação e propõem metodologias para a avaliação educacional. Nessa abordagem se defende a participação dos envolvidos e interessados nos processos avaliativos. A avaliação aparece, por um lado, como possibilidade de se conhecer como foi construída a teoria em avaliação educacional a partir da literatura estadunidense, assim como estão presentes as primeiras tentativas para se pensar uma teoria e prática de avaliação de cunho mais participativo; e por outro lado, evidenciam-se os esforços de estudiosos em esboçar uma prática de avaliação que pudesse ser aplicada à realidade educacional brasileira. 
Essas duas correntes de avaliação também mostram que o campo da avaliação educacional brasileiro na década de 1980 foi habitado por autores de duas diferentes matizes epistemológicas: uma que enfatizava a avaliação em sua vertente de testes e indicadores quantitativos e outra que, contrapondo-se a esse viés quantitativista, ancorados numa abordagem de avaliação qualitativa e participativa, passaram a desenvolver referenciais teóricos alternativos ao modelo de avaliação quantitativo dominante no período. Ainda que antagônicas, essas suas perspectivas apresentam aproximações que indicam a existência de complementaridades em torno da importância da avaliação, da necessidade de qualificação dos avaliadores, da diversificação dos métodos avaliativos e do uso dos resultados para fins de melhoria do que foi avaliado.

Nessa aproximação fica claro que avaliar é uma atividade complexa e multidisciplinar, e que por isso mesmo não pode ser reduzida a uma função mecânica. Trata-se de um ato de poder, e enquanto tal pode ser exercido para a dominação ou para a promoção do bem comum. Exige-se uma formação qualificada para os que realizam as atividades avaliativas, sejam eles professores, agências governamentais, fundações especializadas na aplicação de exames vestibulares, etc. E de que não há um único método a ser aplicado enquanto procedimento de avaliação, este depende do objeto que será avaliado e do que se espera no estudo. Os resultados de uma avaliação deveriam possibilitar a tomada de decisão para fins de melhoramento e redefinição das ações no seu sentido construtivo. O ideal é que os resultados fossem amplamente divulgados junto aos interessados pelo processo: escolas, alunos, pais, sociedade em geral e governos.

\section{Referências}

BARREYRO, Gladys Beatriz; ROTHEN, José Carlos. Para uma história da avaliação da educação superior brasileira: análise dos documentos do Paru, Cnres, Geres e Paiub. Avaliação, Campinas; Sorocaba, v. 13, n. 1, p. 131-152, mar. 2008. Disponível em: http://www.scielo.br/scielo.php?pid=S141440772008000100008\&script=sci_abstract\&tlng=pt. Acesso em: 27 fev. 2018.

BARRETTO, Elba Siqueira de Sá; PINTO, Regina Paim. Avaliação na educação básica, 1990-1998. Brasília: MEC/Inep/Comped, 2001.

BARRETTO, Elba Siqueira de Sá; PINTO, Regina Paim; MARTINS, Angela Maria; DURAN, Marília Claret Geraes. Avaliação na Educação Básica nos anos 90 segundo os periódicos acadêmicos. Cadernos de Pesquisa, São Paulo, n. 114, p. 49-88, nov. 2001. 
BORGES, Regilson Maciel. CALDERÓN, Adolfo Ignacio. Avaliação educacional: o estado do conhecimento da Revista Ensaio: Avaliação e Políticas Públicas em Educação. Ensaio: Avaliação e Políticas Públicas em Educação, Rio de Janeiro v. 19 n. 70, p. 42-56, jan./mar. 2011.

BORGES, Regilson Maciel. Os alicerces da avaliação educacional no Brasil: a elaboração teórica de intelectuais na década de 1980. 2017. Tese (Doutorado em Educação) - Programa de Pós-Graduação em Educação, Universidade Federal de São Carlos, São Paulo, 2017.

BOURDIEU, Pierre. Coisas ditas. São Paulo: Brasiliense, 2004.

CALDERÓN, Adolfo Ignacio; BORGES, Regilson Maciel. La evaluación educacional en el Brasil: de la transferencia cultural a la evaluación emancipadora. Educación, Lima, v. 22, p. 77-95, mar. 2013a.

CALDERÓN, Adolfo Ignacio; BORGES, Regilson Maciel. Avaliação educacional: uma abordagem à luz das revistas científicas brasileiras. Revista Iberoamericana de Evaluación Educativa, Madrid, Espanha, v. 6, n. 1, p. 167-183, mar. 2013 b.

CANDAU, Vera Maria; OSWALD, Maria Luisa M. B. Avaliação no Brasil: uma revisão bibliográfica. Cadernos de Pesquisa, São Paulo, n. 95, p. 25-36, nov. 1995.

DEPRESBITERIS, Léa. Avaliação educacional em três atos. São Paulo: Editora Senac, 1999.

DIAS SOBRINHO, José. Campo e caminhos da avaliação: a avaliação da educação superior no Brasil. In: FREITAS, Luiz Carlos (Org.). Avaliação: construindo o campo e a crítica. Florianópolis: Insular, 2002. p. 13-62.

FREITAS, Dirce Nei Teixeira de. Avaliação da educação básica no Brasil: dimensão normativa, pedagógica e educativa. Campinas: Autores Associados, 2007.

FREZZA, Flávia S.; SILVA, Itamar Mendes. Documentos de Avaliação: análise das produções apresentadas nos Grupos de Trabalho da Associação Nacional de Pós-Graduação em Educação - ANPED - entre 2000 a 2008. In: ENCONTRO DE INICIAÇÃO CIENTÍFICA DA PUC-CAMPINAS, 25., 2009, Campinas. Anais [...] Campinas: PUCCampinas, 2009. p. 1-6.

GAMA, Zacarias Jaegger; COELHO, Daniel Bedirian; CAMELO, Jordan Aragão. Avaliação Educacional: geografia de textos na internet - explorações iniciais. Revista Iberoamericana de Educación, Madrid, v. 42, n. 1, p.1-11, feb. 2007.

GATTI, Bernardete Angelina. Avaliação educacional no Brasil: pontuando uma história de ações. Eccos - Revista Científica, São Paulo, v. 4, n. 1, p. 17-41, jun. 2002.

GATTI, Bernardete Angelina. Avaliação de sistemas educacionais no Brasil. Sísifo - Revista de Ciências da Educação, Lisboa, v. 1, n. 9, p. 7-18, maio/ago. 2009. 
GONÇALVES FILHO, Francisco. Enfoques de avaliação institucional em revista: um estudo da revista Avaliação (1996-2002). 2003. Dissertação (Mestrado em Educação) Faculdade de Educação, Universidade Estadual de Campinas, Campinas, 2003.

HAYASHI, Maria Cristina Piumbato Innocentini et al. História da educação brasileira: a produção científica na biblioteca eletrônica SCIELO. Educação \& Sociedade, Campinas, v. 29, p. 181-211, jan./abr. 2008.

HAYASHI, Carlos Roberto Massao. Apontamentos sobre a coleta de dados em estudos bibliométricos e cientométricos. Filosofia e Educação, Campinas, v. 5, n. 2, p. 89-102, out. 2013.

MEADOWS, Arthur Jack. A comunicação científica. Brasília: Biriquet de Lemos, 1999.

NOSELLA, Paolo. A pesquisa em educação: um balanço da produção dos programas de pósgraduação. Revista Brasileira de Educação, Rio de Janeiro, v. 15, n. 43, p. 177-203, jan./abr. 2010.

PENNA FIRME, Thereza. Avaliação: resposta, responsabilidade, integração. In: BRASIL. Ministério da Educação. Secretaria de Educação Superior. Estudos e confrontos: universidade/ensino de $1^{\circ}$ grau: coletânea de textos sobre os benefícios mútuos de uma integração. Brasília: MEC; Uberlândia: UFU, 1988. p. 135-64.

POLTRONIERI, Heloisa; CALDERÓN, Adolfo Ignacio. Avaliação na educação básica: a revista estudos em avaliação educacional. Estudos em Avaliação Educacional, São Paulo, v. 23, n. 53, p. 82-103, set./dez. 2012.

RICARDO FILHO, Geraldo Sabino. O discurso sobre as políticas educacionais: coesões e ramificações dos especialistas em educação (1990-2007). 2010. Tese (Doutorado em Educação) - Faculdade de Educação, Universidade de São Paulo, São Paulo, 2010.

ROTHEN, José Carlos; BARREYRO, Gladys Beatriz. Avaliação da educação. In: ROTHEN, José Carlos; BARREYRO, Gladys Beatriz. Avaliação da educação: diferentes abordagens críticas. São Paulo: Xamã, 2011a. p. 11-16.

ROTHEN, José Carlos; BARREYRO, Gladys Beatriz. A "RAIES" e a Revista Avaliação a construção de um marco teórico, político e metodológico. Avaliação, Campinas; Sorocaba, v. 16, n. 2, p. 267-290, jul. 2011b. Disponível em: http://www.scielo.br/scielo.php?pid=S141440772011000200003\&script=sci_abstract\&tlng=pt._Acesso em: 27 fev. 2018.

SAUL, Ana Maria. Avaliação emancipatória: desafio à teoria e à prática de avaliação e reformulação de currículo. 8. ed. São Paulo: Cortez, 2010.

SAVIANI, Dermeval. Ensaios contra-hegemônicos: as pedagogias críticas buscando orientar a prática educativa (1980-1991). In: SAVIANI, Dermeval. Histórias das ideias pedagógicas no Brasil. 4. ed. Campinas: Autores Associados, 2013. p. 401-424. 
SILVA, Itamar Mendes da. A discussão sobre avaliação nas reuniões anuais da ANPED no período 2000 a 2010. Avaliação, Campinas; Sorocaba, v. 18, n. 2, p. 335-350, jul. 2013. Disponível em: http://www.scielo.br/scielo.php?pid=S1414-

40772013000200006\&script=sci_abstract\&tlng=pt. Acesso em: 27 fev. 2018.

SOARES, Magda. Alfabetização no Brasil: o estado do conhecimento. Brasília: INEP, 1989.

SOUSA, Clarilza Prado de. Descrição de uma trajetória na/da avaliação educacional. Idéias, São Paulo, n. 30, p. 161-174, 1998.

SOUSA, Sandra Maria Zakia Lian. Avaliação da aprendizagem: natureza e contribuições da pesquisa no Brasil no período de 1980 a 1990. 1994. Tese (Doutorado em Educação) Faculdade de Educação, Universidade de São Paulo, São Paulo, 1994a.

SOUSA, Sandra Maria Zakia Lian. Avaliação da aprendizagem: a divulgação das pesquisas realizadas no âmbito dos programas de pós-graduação. Ensaio: Avaliação e Políticas Públicas em Educação, Rio de Janeiro, v. 1, n. 3, p. 73-79, abr./jun. 1994b.

SOUSA, Sandra Maria Zakia Lian. Avaliação da aprendizagem nas pesquisas do Brasil de 1930 a 1980. Cadernos de Pesquisa, São Paulo, n. 94, p. 43-49, ago. 1995.

SOUSA, Sandra Maria Zakia Lian. Avaliação da aprendizagem: análise das pesquisas produzidas no Brasil, no período de 1980 a 1990. Revista da Faculdade de Educação, São Paulo, v. 22, n. 1, p. 111-144, jan./jun. 1996.

SOUSA, Sandra Maria Zakia Lian. 40 anos de contribuição a avaliação educacional. Estudos em Avaliação Educacional, São Paulo, v. 16, n. 31, p. 7-36, jan./jun. 2005.

SOUSA, Sandra Maria Zakia Lian. Avaliação educacional: tratamento do tema na Rbep no período de 1998 a 2010. Revista Brasileira de Estudos Pedagógicos, Brasília, v. 93, n. 234, p. 372-388, maio/ago. 2012.

STREMEL, Silvana. Aspectos teórico-metodológicos para a análise da constituição do campo acadêmico da política educacional no Brasil. Revista de Estudios Teóricos y

Epistemológicos en Política Educativa, Ponta Grossa, v. 2, n. 1, p. 1-19, ene./jun. 2017.

STUMPF, Ida. Avaliação das revistas de comunicação pela comunidade acadêmica da área. Em Questão, Porto Alegre, v. 9, n. 1, p. 25-38, jan./jun. 2003.

TAVARES, Maria das Graças M., OLIVEIRA, Maria Antonieta A., SEIFFERT, Otília Maria L. B. Avaliação da educação superior na revista Ensaio: Avaliação e Políticas Públicas em Educação: ênfases e tendências. Ensaio: Avaliação e Políticas Públicas em Educação, Rio de Janeiro, v. 19, n. 71, p. 233-258, abr./jun. 2011.

TEIXEIRA, Célia Regina. O "Estado da Arte": a concepção de avaliação educacional veiculada na produção acadêmica do Programa de Pós-Graduação em Educação Currículo (1975-200). Cadernos de Pós-Graduação - educação, São Paulo, v. 5, n. 1, p. 59-66, 2006. 
ULER, Arnilde Martha. Avaliação da aprendizagem: um estudo sobre a produção acadêmica dos programas de pós-graduação em educação (PUCSP, USP, UNICAMP) (20002007). 2010. Tese (Doutorado em Educação: Currículo) - Pontifícia Universidade Católica de São Paulo, São Paulo, 2010.

VIANNA, Heraldo Marelim. Avaliação educacional nos Cadernos de Pesquisa. Cadernos de Pesquisa, São Paulo, n. 80, p. 100-105, 1992. 